\title{
Lower Radiation Dosing in Cardiac CT Angiography: The CONVERGE Registry
}

Paul Madaj ${ }^{1}$, Dong $\mathrm{Li}^{1}$, Rine Nakanishi ${ }^{1,2}$, Daniele Andreini ${ }^{3}$, Gianluca Pontone ${ }^{3}$, Edoardo Conte ${ }^{3}$, Rachael O'Rourke Franzcr $^{4,5}$, Christian Hamilton-Craig ${ }^{4}$, Manojna Nimmagadda ${ }^{1}$, Nicholas Kim ${ }^{1}$, Badiha Fatima ${ }^{1}$, Christopher Dailing ${ }^{1}$, and Matthew J. Budoff ${ }^{1}$

${ }^{I}$ Department of Cardiology, Lundquist Institute, Torrance, California; ${ }^{2}$ Departments of Cardiovascular Medicine and Internal Medicine, Toho University Faculty of Medicine, Tokyo, Japan; ${ }^{3}$ Centro Cardiologico Monzino, IRCCS, Milan, Italy; ${ }^{4}$ Department of Medical Imaging, Prince Charles Hospital, Brisbane, Queensland, Australia; and ${ }^{5}$ University of Queensland, Brisbane, Queensland, Australia

Coronary artery disease is the leading cause of morbidity and mortality. Tools have been developed to accurately diagnose and evaluate coronary artery disease. Coronary CT angiography (CCTA) provides detailed imaging to deliver precise analysis and prognostic information. We sought to compare the radiation dose from a 256-detector-row CT scanner with that from a 64-detector-row CT scanner across a similar profile of patients. Methods: Consecutive patients were screened for the Converge Registry study and, after consenting to be included, were enrolled in accordance with an Institutional Review Board-approved protocol. A control group who underwent 64-row CCTA were matched by age, sex, and body mass index (BMI) with a group who underwent 256-row CCTA. Results: We compared 110 patients in each group. We found that mean dose-length product (DLP) was significantly lower in the 256-row group than in the 64row group $(P<0.05)$. The radiation dose was reduced by $32 \%$ with use of the 256-row scanner for BMls of 18.5-24.9 (DLP, 111.2 vs. $76.1 \mathrm{mGy}-\mathrm{cm}$ [1.56 vs. $1.07 \mathrm{mSv}$; $P<0.05)$. For each BMI subgroup, there was a significant decrease in dose. Regression analysis found that with increasing BMls, DLP significantly increased for both scanners. Conclusion: The 256-row scanner provided CCTA scans at significantly lower radiation doses than the 64-row scanner in different BMI groups, with all other variables accounted for. Lower radiation exposure along with lower contrast requirements can provide images with high diagnostic accuracy and less risk to the patient.

Key Words: cardiac computed tomographic angiography; radiation dosing; radiation safety; coronary artery disease

J Nucl Med Technol 2020; 48:58-62

DOI: $10.2967 /$ jnmt.119.229500

C oronary artery disease is the leading cause of morbidity and mortality around the world. Tools have been developed to accurately diagnose and evaluate coronary artery

Received Apr. 10, 2019; revision accepted Aug. 5, 2019.

For correspondence or reprints contact: Matthew Budoff, Los Angeles

Biomedical Research Institute, 1124 W. Carson St., Torrance CA 90502.

E-mail: mbudoff@labiomed.org

Published online Oct. 11, 2019.

COPYRIGHT (c) 2020 by the Society of Nuclear Medicine and Molecular Imaging. disease, allowing physicians to directly target both atherosclerosis and significant stenosis. Coronary CT angiography (CCTA) scans provide detailed imaging and deliver precise analysis and prognostic information to a clinician and patient. Because of its high negative predictive value for coronary artery disease, CCTA has become a gatekeeper for the assessment of patients with chest pain of recent onset (1). At times, these modalities can be combined with other imaging techniques (e.g., myocardial perfusion scanning or fractional flow reserve) to provide additional diagnostic information, even in the acute setting $(2,3)$. Patients and clinicians are increasingly concerned about the amount of radiation used in medical imaging. With advances in imaging technology and techniques, CCTA can be obtained with more diagnostic information at lower radiation doses (including structural information, along with information about atherosclerosis and obstruction) (4-7). A new wide-volume scanner with 256 detector rows, $16-\mathrm{cm}$ cranial-caudal coverage, and a fast gantry rotation time of $280 \mathrm{~ms}$ (Revolution CT; GE Healthcare) that is now available allows acquisition of the whole heart within a single heartbeat with prospective triggering. Additionally, this scanner uses a new iterative reconstruction algorithm (adaptive statistical iterative reconstruction $\mathrm{V}$ [ASIR-V]) that can allow for lower-milliamperage acquisitions. These technologies allow for lower-dose imaging. CCTA has been shown to be more accurate compared to other imaging modalities such as nuclear or echocardiography imaging for assessment of obstructive disease in both the acute and the outpatient settings (8). HamiltonCraig et al. and Dedic et al. described how the use of CCTA in the acute care setting allows for less outpatient testing and lower medical costs $(9,10)$. We sought to compare the radiation dose from the 256-row scanner with that from a 64-row scanner (LightSpeed VCT; GE Healthcare) across 2 similar groups of patients, matched for age, sex, and size.

\section{MATERIALS AND METHODS}

One hundred ten consecutive patients were screened for the Converge Registry study and, after consenting to be included, were 
enrolled in accordance with an Institutional Review Board-approved protocol. These patients underwent CCTA on the 256-row scanner. A control group of 110 patient matching the test group for age, sex, and body mass index (BMI) underwent CCTA on the 64-row scanner. The scans were conducted at multiple sites including the United States, Italy, and Australia. All studies were read by 2 expert physicians, with adjudication by consensus if there was disagreement on stenosis severity, plaque severity, or image quality.

\section{Patient Preparation}

Certified CCTA technicians performed all the scans. Patients received an oral or intravenous $\beta$-blocker (metoprolol), or both, as needed to achieve a goal heart rate (HR) of less than 70 beats $/ \mathrm{min}$. Individuals presenting with a baseline HR of greater than 65 beats/min were administered oral $\beta$-blocker therapy as the preferred method for slowing the HR. Intravenous administration was used when patients had a persistent HR of greater than 65 beats/min while on the scanner table, using metoprolol at 5-mg increments to a total possible dose of $30 \mathrm{mg}$ to achieve a resting HR of less than 65 beats/min.

\section{Acquisition Protocol}

Contrast Administration. After a scout radiograph of the chest (anteroposterior and lateral) had been obtained, a timing bolus (using $10-20 \mathrm{~mL}$ of contrast medium) was performed to determine the time to optimal contrast opacification in the axial image at a level immediately superior to the ostium of the left main coronary artery. Sublingual nitroglycerin $(0.4 \mathrm{mg})$ was given immediately before the contrast injection. During the CCTA acquisition, $80 \mathrm{~mL}$ of iodinated contrast medium (Visipaque; GE Healthcare) was injected using a triple-phase protocol: $60 \mathrm{~mL}$ of iodixanol, followed by $40 \mathrm{~mL}$ of a 50:50 mixture of iodixanol and saline, followed by a $50-\mathrm{mL}$ saline flush.

64-Row Acquisition. The 64-row acquisition was performed with prospective gating, using a commercially available protocol (SnapShot Pulse, GE Healthcare) and the following parameters: sixty-four $0.625-$ mm slices, the smallest $\mathrm{x}$-ray window (only $75 \%$ of the R-R interval), a $z$-axis coverage of $40 \mathrm{~mm}$ with an increment of $35 \mathrm{~mm}$, a gantry rotation time of $350 \mathrm{~ms}$, tube voltage of $120 \mathrm{mV}$, and an effective milliamperage of 350-780 mA. The field of view was $25 \mathrm{~cm}$. Scanning was performed from $10 \mathrm{~mm}$ above the origin of the left main coronary artery (determined on a calcium scan) to the diaphragm. By choosing the smallest possible window at only 1 distinct end-diastolic phase of the R-R interval (i.e., 75\%), we ascertained the lowest effective dose delivery achievable. The amount of radiation used to evaluate both intensity and scan length was registered in DLP $(\mathrm{mGy}-\mathrm{cm})$ and was later converted to millisieverts using a factor of 0.014 .

256-Row Acquisition. The 256-row scanner is a volumetric device that has high-definition spatial resolution and a $16-\mathrm{cm}$ detector array. The field of view ( $z$-axis) included the mid-ascending aorta to the upper abdomen. No table movement occurred during axial volumetric scanning because of the $16 \mathrm{~cm}$ of $z$-axis coverage, and no patient required more than $16 \mathrm{~cm}$ of $z$-axis coverage. Selection of the $z$-axis collimation was based on the scout images demonstrating the heart size. Tube voltage was fixed at $120 \mathrm{kVp}$, to provide comparable radiation between scans and scanners. Tube current ranged from 122 to $740 \mathrm{~mA}$. A medium field of view $(25 \mathrm{~cm})$ was selected for all patients. The gantry rotation time was $0.28 \mathrm{~s}$, with a minimum temporal resolution of $140 \mathrm{~ms}$. The scanner is equipped with autogating capability, which automatically adjusts
HR-dependent settings for triggered acquisition and gated reconstruction. Autogating was used to automatically acquire diastolic phases for lower HRs and both systolic and diastolic phases for higher HRs. Electrocardiographic dose modulation, which reduces the milliamperage for nontarget phases, was used in the higher-HR acquisitions. All acquisitions were prospectively gated. Approximately $50-80 \mathrm{~mL}$ of contrast medium was used. Each scan was done in a 1-heartbeat acquisition within 1 cardiac cycle, regardless of the HR. Motioncorrection software (SnapShot Freeze; GE Healthcare) was used to correct motion artifacts in patients with higher HRs. The amount of radiation used to evaluate both intensity and scan length was registered in DLP (mGy-cm) and was later converted to millisieverts using a factor of 0.014 .

\section{Image Reconstruction}

Thin $(0.625-\mathrm{mm})$ image reconstruction was used, with intervals ranging from $60 \%$ to $80 \%$; most data were reconstructed at $75 \%$ of the R-R interval. Fifty percent ASIR-V was used. The images were transferred to an external workstation (Advantage Workstation, version 4.4; GE Healthcare) for interpretation.

\section{Statistical Analysis}

Regression analysis and $t$ testing were used to compare the test group with the control group. Further analysis was also performed on subgroups of clinical variables within the test group.

\section{RESULTS}

The mean DLP was significantly lower in the 256-row test group than in the 64-row control group $(P<0.05)$ (Table 1). Use of the 256-row scanner reduced the radiation dose by $32 \%$ for BMIs of 18.5-24.9 (DLP, 111.2 vs. $76.1 \mathrm{mGy}-\mathrm{cm}$ [1.56 vs. $1.07 \mathrm{mSv}$ ]; $P<0.05$ ). For each BMI subgroup, there was a significant decrease in dose for the 256-row scanner as compared with the 64-row scanner. Regression analysis found that with increasing BMIs (Table 2), DLP (and radiation exposure) significantly increased for both scanners for BMIs of 18.5-24.9, compared with BMIs of 25-29.9 or BMIs of greater than 30 (all $P<0.05$ ).

\section{DISCUSSION}

The 256-row scanner provided CCTA scans at lower radiation doses than the 64-row scanner, with all other variables controlled for (sex, age, and BMI). As previous studies have demonstrated, with improved technology, lower radiation exposure along with lower contrast requirements can provide quality imaging with high diagnostic accuracy and less risk to the patient $(11,12)$. The 256-row scanner allows improved image quality and clinical capabilities through the convergence of coverage, spatial resolution, and temporal resolution advantages over the 64-row scanner. The rotation speed is faster (280 ms, vs. $350 \mathrm{~ms}$ with the 64-row scanner), reducing patient exposure by at least $20 \%$ (Table 1). Furthermore, the whole-heart coverage allows the heart to be imaged in a single rotation ( 1 heartbeat) because of the $16-\mathrm{cm} z$-axis coverage with no table movement, as compared with the 5heartbeat acquisition of the 64-row scanner (which has z-axis coverage of only $4 \mathrm{~cm}$ ). Improved imaging protocols and technology can aid in obtaining adequate imaging despite 
TABLE 1

Demographic and Clinical Characteristics

\begin{tabular}{lccc}
\hline \multicolumn{1}{c}{ Characteristic } & Control group $(n=110)$ & Test group $(n=110)$ \\
\hline Age $(\mathrm{y})$ & $60.2 \pm 12.5$ & $61.1 \pm 12.5$ & $P$ \\
Female $(n)$ & $34(31.5 \%)$ & $35(31.8 \%)$ & 0.615 \\
Weight $(\mathrm{kg})$ & $83.5 \pm 17.0$ & $83.3 \pm 17.1$ & 0.957 \\
BMI & $28.7 \pm 6.1$ & $27.2 \pm 4.4$ & 0.949 \\
Total DLP (for 110 patients) (mGy-cm) & $141.0 \pm 78.8(1.97 \pm 1.10 \mathrm{mSv})$ & $113.5 \pm 53.6(1.59 \pm 0.75 \mathrm{mSv})$ & 0.326 \\
DLP stratified by BMl (mGy-cm) & & & 0037 \\
Normal weight (BMI, 18.5-24.9) & $111.2 \pm 84.7(1.56 \pm 1.19 \mathrm{mSv})$ & $76.1 \pm 49.0(1.07 \pm 0.69 \mathrm{mSv})$ & 0.044 \\
Overweight (BMI, 25-29.9) & $133.0 \pm 55.1(1.86 \pm 0.77 \mathrm{mSv})$ & $112.4 \pm 52.1(1.57 \pm 0.73 \mathrm{mSv})$ & 0.047 \\
Obese (BMI, $>30)$ & $169.2 \pm 74.1(2.37 \pm 1.04 \mathrm{mSv})$ & $142.3 \pm 28.9(1.99 \pm 0.40 \mathrm{mSv})$ & 0.0004
\end{tabular}

Values are expressed as mean $\pm S D$, except for age.

large body habitus or arrhythmias. Zhao et al. showed that CCTA images can be evaluated with high diagnostic accuracy even in patients with arrhythmias (e.g., atrial fibrillation) (13). In addition, the dose reduction is independent of iterative dose-reduction algorithms. ASIR-V allows for more advanced modeling because it deemphasizes the system optics modeling, enabling reconstruction speeds similar to those of filtered backprojection. Because ASIR-V was used on both systems, the dose reduction associated with use of 256-row scanning is incremental to this technique (14). With the new-generation 256-row CT scanner along with accompanying protocols, we are able to perform CCTA at significantly lower radiation doses.

Physicians' and patients' concerns about radiation doses should be alleviated. Studies have shown that CCTA images can be acquired with as little as $1.1 \pm 0.4 \mathrm{mSv}$ (15-17). Studies by Schmermund et al. and Dogan et al., along with multiple other studies, were able to demonstrate a reduction in CCTA radiation dose over a 5-y period, attributed to a combination of improvements in data acquisition protocols and patient preparation, as well as installation of new CT scanners with advanced technology (18-20).

The use of CCTA has the potential to significantly alter the management of coronary artery disease. An American Heart Association statement on assessment of coronary artery disease by CCTA provides a Class IIa recommendation for the use of CCTA in the assessment of obstructive disease in symptomatic patients (21). Studies have shown CCTA to be the most accurate of the noninvasive imaging modalities in detecting obstructive coronary artery disease, especially when compared with functional tests $(22,23)$. In the multicenter PROMISE study, the prevalence of normal test results and the incidence of cardiac events were significantly lower in patients randomized to CCTA than in patients randomized to functional testing (33.4\% vs. $78.0 \%$ and $0.9 \%$ vs. $2.1 \%$, respectively; both $P<0.001$ ) (24). In a study by Lee et al., after adjustment for confounding risk factors, obstructive coronary artery disease remained an independent predictor of major adverse cardiac events (hazard ratio, 3.11 [95\% confidence interval, 2.00-4.86]; $P<0.001]$ ). Their prediction model for detecting adverse events improved significantly (C-index, 0.788 [95\% confidence interval, 0.747-0.829]; $P=$ 0.0349) when adjusted for traditional risk factors (e.g., age, male, hypertension, hyperlipidemia, smoking, estimated glomerular filtration rate, and hemoglobin $\mathrm{A}_{1 \mathrm{c}}$ ) (25). The 2010 Appropriate Use Criteria for Cardiac CT list CCTA as an appropriate modality in the evaluation of patients with a low to intermediate pretest probability of coronary artery disease, along with evaluation after CABG and structural disease (26). The current study and others demonstrate that the prognostic and diagnostic information can be obtained at lower radiation doses without compromising image quality irrespective of HR, heart rhythm, or obesity (27).

Multiple trials have consistently shown the safety of using a negative CCTA result as a criterion for discharging patients with a low-to-intermediate pretest probability of coronary artery disease from the emergency department. Such patients

TABLE 2

Association of DLP Levels and BMI Between Test Group and Control Group

\begin{tabular}{lrrr}
\hline \multicolumn{1}{c}{ BMI } & DLP $(\mathrm{mGy}-\mathrm{cm})(n=110)$ & $\beta$ & 95\% confidence interval \\
\hline $18.5 \leq \mathrm{BMI} \leq 24.9$ & $76.1 \pm 49.0(1.07 \pm 0.69 \mathrm{mSv})$ & 0 (reference) & \\
$25 \leq \mathrm{BMl} \leq 29.9$ & $112.4 \pm 52.1(1.57 \pm 0.73 \mathrm{mSv})$ & $28.3(\mathrm{SE}, 11.1)$ & $6.5-50.1$ \\
$30 \leq \mathrm{BMI}$ & $142.3 \pm 28.9(1.99 \pm 0.40 \mathrm{mSv})$ & $53.8(\mathrm{SE}, 11.9)$ & $28.2-75.8$
\end{tabular}

${ }^{*}$ Adjusted for age and sex.

DLP values are expressed as mean \pm SD. 
subsequently have low rates of major adverse cardiovascular events and are discharged sooner and at significantly lower cost (28). Meyersohn et al. demonstrated a significant reduction in radiation dose with higher-generation $\mathrm{CT}$ scanners in the emergency room setting (29). With these improvements in technology allowing for greater volume coverage with single acquisitions, centers can reduce the radiation dose and the amount of contrast medium given to a patient during a CCTA acquisition. For example, Van Cauteren et al. demonstrated up to a 50\% reduction in iodine dose (30). Doses can go even lower with use of a lower kilovolt potential, further reducing doses well below background radiation levels and even lower than for calcium scoring, which is fixed at $120 \mathrm{kVp}(31,32)$. One does not need to alter scanning techniques to obtain similar image quality at lower radiation doses $(33,34)$.

A limitation of our study is that rather than scanning the same patients with both protocols, we chose to match the 256-row test group to a 64-row control group by age, sex, and BMI to create approximately similar cohorts. More prospective studies using larger sample sizes need to be conducted to further study CCTA radiation doses.

\section{CONCLUSION}

Compared with the 64-row scanner, the 256-row scanner provided CCTA scans with more clinically relevant information at significantly lower radiation doses, enabling clinicians to make appropriate clinical diagnoses and decisions, and alleviating patient concerns about radiation.

\section{DISCLOSURE}

Matthew Budoff receives grant support from the National Institutes of Health and GE Healthcare. GE Healthcare provided funding to collect scans to create the Converge Registry but had no input on the science or manuscript preparation. No other potential conflict of interest relevant to this article was reported.

\section{REFERENCES}

1. Treibel TA, Rossi A, Pugliese F, Davies LC. Functional assessment of coronary artery disease by cardiac computed tomography. Expert Rev Cardiovasc Ther. 2017;15:657-665.

2. Han R, Sun K, Lu B, Zhao R, Li K, Yang X. Diagnostic accuracy of coronary CT angiography combined with dual-energy myocardial perfusion imaging for detection of myocardial infarction. Exp Ther Med. 2017;14:207-213.

3. Kim KH, Doh JH, Koo BK, et al. A novel noninvasive technology for treatment planning using virtual coronary stenting and computed tomography-derived computed fractional flow reserve. JACC Cardiovasc Interv. 2014;7:72-78.

4. Koplay M, Erdogan H, Avci A, et al. Radiation dose and diagnostic accuracy of high-pitch dual-source coronary angiography in the evaluation of coronary artery stenoses. Diagn Interv Imaging. 2016;97:461-469.

5. Selçuk T, Otcu H, Yuceler Z, et al. Effectiveness of using dual-source CT and the upshot it creates on both heart rate and image quality. Balkan Med J. 2016;33: 283-293.

6. Hubbard L, Ziemer B, Lipinski J, et al. Functional assessment of coronary artery disease using whole-heart dynamic computed tomographic perfusion. Circ Cardiovasc Imaging. 2016;9:e005325.

7. Hou Y, Ma Y, Fan W, et al. Diagnostic accuracy of low-dose 256-slice multidetector coronary CT angiography using iterative reconstruction in patients with suspected coronary artery disease. Eur Radiol. 2014;24:3-11.
8. Budoff M. The role of cardiac computed tomography in the evaluation of patients with stable ischemic heart disease. American College of Cardiology website. https://www.acc.org/latest-in-cardiology/articles/2016/02/05/13/46/the-role-of-cctin-the-evaluation-of-patients-with-sihd. Published February 8, 2016. Accessed November 6, 2019.

9. Hamilton-Craig C, Fifoot A, Hansen M, et al. Diagnostic performance and cost of CT angiography versus stress ECG: a randomized prospective study of suspected acute coronary syndrome chest pain in the emergency department (CTCOMPARE). Int J Cardiol. 201420;177:867-873.

10. Dedic A, Lubbers MM, Schaap J, et al. Coronary CT angiography for suspected ACS in the era of high-sensitivity troponins: randomized multicenter study. $J \mathrm{Am}$ Coll Cardiol. 2016;67:16-26.

11. Wang W, Zhao YE, Qi L, et al. Prospectively ECG-triggered high-pitch coronary $\mathrm{CT}$ angiography at $70 \mathrm{kVp}$ with $30 \mathrm{~mL}$ contrast agent: an intraindividual comparison with sequential scanning at $120 \mathrm{kVp}$ with $60 \mathrm{~mL}$ contrast agent. Eur J Radiol. 2017;90:97-105.

12. Magnacca M, Poddighe R, Del Meglio J, et al. Teamwork for cardiac imaging: coronary computed tomography angiography and low-dose radiation exposure: a cardiology center experience [in Italian]. G Ital Cardiol (Rome). 2017;18:313-321.

13. Zhao C, Jiang M, Liu J, Gao L, Wang X. Feasibility of low-dose coronary computed tomographic angiography used in atrial fibrillation patients. Acta Cardiol. 2017;72:292-298.

14. Sulaiman N, Soon J, Park JK, et al. Comparison of low-dose coronary artery calcium scoring using low tube current technique and hybrid iterative reconstruction vs. filtered back projection. Clin Imaging. 2017;43:19-23.

15. Andreini D, Mushtaq S, Conte E, et al. Coronary CT angiography with $80 \mathrm{kV}$ tube voltage and low iodine concentration contrast agent in patients with low body weight. J Cardiovasc Comput Tomogr. 2016;10:322-326.

16. Tsay IM, Subiakto I, Asrar Ul Haq M, et al. Radiation dose difference between state of the art myocardial perfusion scintigraphy and computed tomography coronary angiography in patients undergoing evaluation for suspected coronary artery disease. Intern Med J. 2016;46:226-229.

17. Lee JW, Kim CW, Lee HC, et al. High-definition computed tomography for coronary artery stents: image quality and radiation doses for low voltage (100 $\mathrm{kVp})$ and standard voltage $(120 \mathrm{kVp})$ ECG-triggered scanning. Int J Cardiovasc Imaging. 2015;31(suppl 1):39-49.

18. Schmermund A, Marwan M, Hausleiter J, et al. Declining radiation dose of coronary computed tomography angiography: German cardiac CT registry experience 2009-2014. Clin Res Cardiol. 2017;106:905-912.

19. Dogan N, Dursun A, Ozkan H, Karatas S, Celiloglu N, Agca FV. Low radiation dose computed tomography coronary angiography: evaluation of the variations in coronary arteries. Surg Radiol Anat. 2016;38:1123-1134.

20. Kazakauskaite E, Husmann L, Stehli J, et al. Image quality in low-dose coronary computed tomography angiography with a new high-definition CT scanner. Int J Cardiovasc Imaging. 2013;29:471-477.

21. Budoff MJ, Achenbach S, Blumenthal RS, et al. Assessment of coronary artery disease by cardiac computed tomography: a scientific statement from the American Heart Association Committee on Cardiovascular Imaging and Intervention, Council on Cardiovascular Radiology and Intervention, and Committee on Cardiac Imaging, Council on Clinical Cardiology. Circulation. 2006;114:1761-1791.

22. Patel MR, Dai D, Hernandez AF, et al. Prevalence and predictors of nonobstructive coronary artery disease identified with coronary angiography in contemporary clinical practice. Am Heart J. 2014;167:846-852.e2.

23. Neglia D, Rovai D, Caselli C, et al. Detection of significant coronary artery disease by noninvasive anatomical and functional imaging. Circ Cardiovasc Imaging. 2015;8:e002179.

24. Hoffmann U, Ferencik M, Udelson JE, et al. Prognostic value of noninvasive cardiovascular testing in patients with stable chest pain: insights from the PROMISE trial (Prospective Multicenter Imaging Study for Evaluation of Chest Pain). Circulation. 2017;135:2320-2332.

25. Lee KY, Hwang BH, Kim TH, et al. Computed tomography angiography images of coronary artery stenosis provide a better prediction of risk than traditional risk factors in asymptomatic individuals with type 2 diabetes: a long-term study of clinical outcomes. Diabetes Care. 2017;40:1241-1248.

26. Taylor AJ, Cerqueira M, Hodgson JM, et al. ACCF/SCCT/ACR/AHA/ASE/ ASNC/NASCI/SCAI/SCMR 2010 Appropriate use criteria for cardiac computed tomography: a report of the American College of Cardiology Foundation appropriate use criteria task force, the Society of Cardiovascular Computed Tomography, the American College of Radiology, the American Heart Association, the American Society of Echocardiography, the American Society of Nuclear Cardiology, the North American Society for Cardiovascular Imaging, the Society for Cardiovascular Angiography and Interventions, and the Society for Cardiovascular Magnetic Resonance. Circulation. 2010;122:e525-e555. 
27. Ochs MM, Siepen FAD, Fritz T, et al. Limits of the possible: diagnostic image quality in coronary angiography with third-generation dual-source CT. Clin Res Cardiol. 2017;106:485-492.

28. Cury RC, Budoff M, Taylor AJ. Coronary CT angiography versus standard of care for assessment of chest pain in the emergency department. J Cardiovasc Comput Tomogr. 2013;7:79-82.

29. Meyersohn NM, Szilveszter B, Staziaki PV, et al. Coronary CT angiography in the emergency department utilizing second and third generation dual source CT. J Cardiovasc Comput Tomogr. 2017;11:249-257.

30. Van Cauteren T, Van Gompel G, Tanaka K, et al. The impact of combining a lowtube voltage acquisition with iterative reconstruction on total iodine dose in coronary CT angiography. BioMed Res Int. 2017;2017:2476171.
31. Alani A, Nakanishi R, Luo Y, et al. Radiation dose reduction in cardiac CT angiography by applying a low tube voltage: a comparison among 120, 100 and 80 kVp protocols. Curr Med Imaging Rev. 2015;11:192-199.

32. Messenger B, Li D, Nasir K, Carr JJ, Blankstein R, Budoff MJ. Coronary calcium scans and radiation exposure in the multi-ethnic study of atherosclerosis. Int J Cardiovasc Imaging. 2016;32:525-529.

33. Tatsugami F, Higaki T, Fukumoto W, et al. Radiation dose reduction for coronary artery calcium scoring at 320-detector CT with adaptive iterative dose reduction 3D. Int J Cardiovasc Imaging. 2015;31:1045-1052.

34. Willemink MJ, den Harder AM, Foppen W, et al. Finding the optimal dose reduction and iterative reconstruction level for coronary calcium scoring. J Cardiovasc Comput Tomogr. 2016;10:69-75. 